Revue de l'association française de recherche sur

I'histoire du cinéma

$91 \mid 2020$

Ivresse patrimoniale ? - Décors d'Intolérance -

Germaine Dulac - Alice au pays des merveilles - Éric

Rondepierre

\title{
Jean-Pierre Esquenazi, le Dictateur de Charlie Chaplin
}

\section{Francis Bordat}

\section{OpenEdition}

Journals

Édition électronique

URL : https://journals.openedition.org/1895/8095

DOI : 10.4000/1895.8095

ISSN : 1960-6176

Éditeur

Association française de recherche sur l'histoire du cinéma (AFRHC)

Édition imprimée

Date de publication : 1 juin 2020

Pagination : 193-196

ISBN : 978-2-37029-091-5

ISSN : 0769-0959

Référence électronique

Francis Bordat, « Jean-Pierre Esquenazi, le Dictateur de Charlie Chaplin», 1895. Mille huit cent quatrevingt-quinze [En ligne], 91 | 2020, mis en ligne le 10 mai 2021, consulté le 01 juin 2021. URL : http:// journals.openedition.org/1895/8095; DOI : https://doi.org/10.4000/1895.8095

Ce document a été généré automatiquement le 1 juin 2021.

(c) $\mathrm{AFRHC}$ 


\title{
Jean-Pierre Esquenazi, le Dictateur de Charlie Chaplin
}

\author{
Francis Bordat
}

\section{RÉFÉRENCE}

Jean-Pierre Esquenazi, le Dictateur de Charlie Chaplin, Lyon, Presses universitaires de Lyon, Coll. « Le Vif du sujet », 2020, 162 p.

1 Après ceux de Christian Delage (Chaplin : la grande histoire, Jean-Michel Place, 1998) et de Jean Narboni (Pourquoi les coiffeurs? Notes actuelles sur Le Dictateur, Capricci, 2010), voici le troisième livre français consacré en vingt ans à The great Dictator (le Dictateur) de Chaplin, ce qui atteste l'attention particulière qu'on lui porte en France, par contraste avec la relative indifférence critique dont il reste l'objet outre-Manche et outreAtlantique. Signe encore de cette spécificité : une importante exposition à venir (dont la Covid-19 a retardé l'ouverture) consacrée à des archives inédites, notamment photographiques, de la préparation, du tournage et de la réception du film de 1940. Exposition réalisée (pour Lille et Arles) par Sam Stourdzé, Mathilde Thibault-Starzyk et Kate Guyonvarch avec la participation du Chaplin Office.

2 L'ouvrage paraît dans une collection «qui s'intéresse aux œuvres qui, par invention formelle, rupture esthétique ou risque politique, ont su déplacer notre regard sur le monde, sur le cinéma et sur l'art». Rien donc de plus justifié, puisque la question «Comment faire un film politique? » constitue le fil directeur du livre. Elle est reprise sous des angles divers à la fin de chacun de ses cinq chapitres : il s'agit de prendre la pleine mesure de l'engagement du cinéaste dans la cause qu'il embrasse, mais aussi de décrire l'art comique et l'ensemble des moyens artistiques que Chaplin mobilise à ses fins.

3 Même si on en convient mieux aujourd'hui (car comme le notait Narboni, la nouveauté et l'audace du Dictateur "paraissent [...] plus éclatantes à mesure que les années passent », op. cit., p. 13), Esquenazi a raison d'insister sur l'énorme courage qu'il a fallu à 
Chaplin pour mener son projet à bien, face à l'hostilité à peu près générale de Hollywood, de l'Amérique (résolument anti-interventionniste quand commence la production du film en janvier 1939 -les États-Unis ne rentrent en guerre que deux ans plus tard) et de la plupart des diplomaties mondiales (y compris le Foreign office) qui ont tout fait pour l'empêcher. Aujourd'hui encore, la majorité des spectateurs français (j'ai souvent fait le test auprès de mes étudiants) pensent que le Dictateur a été réalisé après la guerre, c'est-à-dire au moment où il a été effectivement distribué en France, ce qui conduit à sous-estimer les risques considérables, aussi bien personnels que financiers, pris par le cinéaste, mais également le caractère extraordinairement prophétique de son propos. Exemple le plus frappant : même si Chaplin écrit dans son autobiographie qu'il aurait hésité à tourner le Dictateur s'il avait pu imaginer l'horreur de la Shoah, il est saisissant de constater que le projet d'extermination des Juifs est annoncé dans le film par Hynkel et ses acolytes avec la plus grande clarté : «Nous exterminerons les Juifs, anéantirons les Bruns! Alors émergera notre rêve, une pure race aryenne ». Chaplin avait bien lu Mein Kampf, et le prenait au sérieux. Esquenazi : "Quelle œuvre artistique aura été aussi explicite à cet instant tragique de notre monde?»

4 Dans le contexte hollywoodien de l'époque, c'était un défi supplémentaire de faire explicitement de Charlot (en la personne de son avatar le Barbier) un Juif. La judéité de Chaplin reste à ce jour sujet de discussion. Tout en disant qu'il n'était pas juif, mais en refusant aussi de dénier qu'il le fût face à ceux qui l'en "accusaient », le comédiencinéaste avait coutume de préciser qu' i il aurait été fier de l'être ». En revanche, pour qui regarde bien ses films, la judéité du personnage de Charlot ne peut faire aucun doute. Comme l'écrivait Hannah Arendt, c'est d'ailleurs « dans ce petit juif, délaissé et inventif, suspecté du monde entier, que le petit homme de toutes les nations se reconnut ». De là cependant à faire de son héros un membre de la communauté du Ghetto, et laisser peindre le mot «JEW » sur les vitres de son échoppe, c'était un pas que n'aurait franchi en 1939 aucun autre producteur hollywoodien s'il avait eu en mains (Heaven forbid!) les destinées de Charlot, alors même que, ou en l'occurrence parce que, les patrons des studios étaient presque tous juifs eux-mêmes. Seule l'indépendance financière de Chaplin (qui était son propre producteur et son propre distributeur) lui a permis de rompre le tabou hollywoodien avec cette revendication affichée de la judéité du Barbier.

5 Le relatif discrédit critique qu'a connu le film - que son succès populaire a fini par faire oublier - a porté sur une question qui concerne l'ensemble du film, mais que sa séquence finale a d'emblée radicalisée.

6 Il s'agit comme on le sait d'un discours de quatre minutes adressé à l'humanité, filmé en plan rapproché fixe. Dans une « espèce de psychanalyse photographique de Charlot [qui] reste certainement l'un des hauts moments du cinéma universel » (André Bazin), on y voit, au fur et à mesure que la parole monte en puissance, les traits de Hynkel se dissoudre dans ceux du Barbier, puis ceux du Barbier dans ceux de Charlot et enfin ceux de Charlot dans ceux de Chaplin lui-même jusqu'à ce que la voix seule du cinéaste, comme céleste et désincarnée, imbrique l'appel au monde et l'invocation à la femme aimée (Hannah, à la fois l'héroïne du film qu'interprète Paulette Goddard et le prénom de la mère de Chaplin). Or cette séquence a été l'objet d'un rejet critique à peu près unanime à la sortie du film et même longtemps après. Charles Maland l'a rappelé : « Si quelque chose comme un consensus critique peut être dégagé des comptes rendus [du 
Dictateur], c'est que, bien que le film soit important et qu'il ne doive pas être manqué, la fin fait long feu et est une erreur " (Chaplin and American Culture: The Evolution of a Star Image, Princeton University Press, p. 182, cité et traduit par Esquenazi, p. 131).

7 Si jadis Jacques Siclier ou Henri Langlois, avant Bazin lui-même, avaient commencé à débouter ces jugements, le dernier chapitre du livre d'Esquenazi en propose la réfutation la plus systématique en contestant méthodiquement les présupposés qui les sous-tendent. Il en compte trois : le discours du Barbier ne respecterait pas le «mode générique " du film; son "contrat fictionnel» se trouverait rompu; il serait "politiquement naïf et maladroit par rapport aux conditions historiques dont il s'inspire ».

8 Mais c'est plus généralement (je veux dire, au-delà de la seule considération du discours final) que la critique "sérieuse " s'est montrée rétive face au «mélange des genres » ainsi qu'au décalage ressenti entre la légèreté de la comédie et la gravité de l'Histoire. L'article du Monde à la sortie du film en France (12 avril 1945) est significatif à cet égard, pour ne pas dire caricatural : "Il est impossible de ne pas être frappé de l'indigence de pensée que révèle le scénario du Dictateur. La personnalité de celui-ci est esquissée en traits tellement grossiers, la bouffonnerie côtoie à un tel point l'invraisemblance et, parallèlement, les rappels historiques sont tellement dénués d'ampleur et de vérité que la cassure se fait de plus en plus nette et de plus en plus profonde, tandis que se déroulent les images, entre la sensibilité du spectateur et les personnages de l'écran ». En 1989 encore, le même journal, tout en reconnaissant que Garbitsch programme effectivement la "solution finale " (avant qu'elle ait été concrètement mise en application par les nazis, souligne justement Esquenazi), considère que «l'éventualité d'une extermination est annulée par la charge grotesque ».

9 On comprend au contraire en lisant ce livre comment la confrontation des genres et des styles - et une "grammaire visuelle " qui emprunte à toutes les sources du rire, du burlesque le plus "grossier» aux ressorts les plus fins et les plus élégants de la comédie - est indissociable du propos lui-même, et d'un enjeu proprement politique qu'Esquenazi compare à juste titre à celui de la satire ménippée. Un point fort de sa démonstration est cette idée que les personnages de Hynkel et du Barbier ne ressortissent pas à la même esthétique - et pas seulement à deux traitements différents du décor et de la prise de vues, comme j'y avais insisté jadis dans mon Chaplin cinéaste. La caricature de Hynkel et des autres pantins qui fréquentent l'univers du «Palais » (par opposition au Barbier et aux autres personnages qui habitent celui du "Ghetto ») puiserait au registre du cinéma «des attractions » qui caractérise encore le premier slapstick sennettien : celui dont le cinéma de Chaplin s'est progressivement détaché en faveur d'un comique plus psychologique, plus construit et globalement plus elliptique qui caractérise son art le plus spécifique et le plus profond: "Afin de proposer une mise en scène propre à exposer le système de puissance hynkelien, afin d'offrir une figuration de la tyrannie à l'ère moderne, Chaplin revient aux attractions. Ce format de mise en scène, qui privilégie le face à face entre l'acteur grimaçant et le spectateur interpellé, qui réduit le cadre à ses dimensions physiques, qui exclut (ou tend à exclure) la possibilité de développement d'un champ comme cadre expressif d'un univers fictionnel, devient le vecteur par lequel le cinéaste exhibe le fascisme " (Esquenazi remarque à ce propos que dans To Be or Not to Be (Jeux dangereux, 1941), Ernst Lubitsch révèle identiquement l'incapacité native des nazis à «entrer dans le tissu des jours ». 
J'aime beaucoup cette formule, qui décrit fort bien aussi le monde du Ghetto selon Chaplin).

10 Hynkel et le Barbier appartiennent à deux univers séparés qui relèveraient également de deux « idiolectes de cinéma ", dont la temporalité même s'avère contradictoire, car si les bonheurs et les inquiétudes animent le Ghetto au rythme de la vraie vie, "l'intermittence, principe temporel du cinéma des attractions », caractérise l'univers paranoïaque et mortifère du premier. Hynkel ne cesse d'ailleurs «de regarder sa montre, comme si le temps était compté : mais il est surtout démembré ».

11 C'est à cet éclairage qu'Esquenazi reprend l'analyse des deux grandes séquences musicales du Barbier et de Hynkel : la séquence du coiffeur sur la Danse hongroise $n^{\circ} 5$ de Brahms et la séquence de la mappemonde sur le prélude de l'Acte 1 du Lohengrin de Wagner. Il repère dans la "pavane du dictateur " «le narcissisme emphatique de la danse, la boursouflure alanguie et pourtant méthodique des poses»; il note au contraire dans la "farandole du Barbier» la "manière de saisir une occasion du quotidien ", la «spontanéité du jeu, « la sobriété de la mise en scène et de la lumière ». S'opposent alors «au rêve de mort du tyran" et à sa "jouissance frénétique et solitaire » «l'utopie heureuse d'une vie dansée ».

12 Mettons un léger bémol à cette utile structure : on ne saurait attacher strictement un contenu à une forme. Comme Esquenazi le signale lui-même, le prélude de Lohengrin accompagne aussi bien la fin du discours humaniste du Barbier que la danse paranoïaque du tyran. Quant au personnage de «l'éternel Charlot », jusqu'en ses alterego du King in New York (Un roi à New York, 1957) ou de A Countess from Hong-Kong (la Comtesse de Hong Kong, 1967), il se nourrit autant des caractères les plus grossiers (agressifs, scatologiques, lubriques) du slapstick originel que des gags plus élaborés et plus subtils du comique chaplinien. L'opposition des personnages et de leur style « de base » ne doit pas conduire à hiérarchiser des formes " inférieures » ou "supérieures » de la comédie. La spécificité du génie chaplinien reste dans la combinaison de ces divers registres, qu'il sollicite identiquement d'un bout à l'autre de son œuvre.

Par ailleurs, si tout le monde peut s'accorder sur le fait que, malgré leur moustache commune, "Hitler est un clown bien inférieur à Charlot ", on ne saurait dire la même chose de Hynkel. Car ce dernier n'est évidemment pas moins drôle que le Barbier, et il n'hérite pas moins que son sosie du génie comique de Charlot - dont il reproduit d'ailleurs quantité de gestes et de comportements - du petit trébuchement cocasse au rafraîchissement de l'entrejambe par un verre d'eau. Esquenazi confirme d'ailleurs ce fait dans un rapprochement pertinent : lorsque Hynkel arrache les boutons de la veste de Herring, «sa frénésie s'apparente à celle du Vagabond vissant tout ce qui ressemble à un écrou à la fin de la séquence de l'usine des Temps modernes ".

14 À signaler enfin l'analyse originale du commandant Schultz incarné par Reginald Gardiner. L'ambiguïté de ce personnage (bien repérée déjà par Narboni) est indispensable à la fois au développement du récit et au message du film. Ses interventions sauvent la vie du Barbier (qui l'a lui-même sauvé pendant la Première Guerre mondiale), mais Schultz encourage ensuite les Juifs du Ghetto à tirer au sort pour se sacrifier dans un attentat suicide (auquel lui-même, noblesse oblige, ne saurait candidater), suite à quoi il trahit son équivocité en lançant (et refoulant aussitôt) un «Heil Hynkel!» réflexe. Or la duplicité, sans doute inconsciente, mais bien réelle quand même, du personnage, résonne à la fois dans l'œuvre chaplinienne et dans la grande Histoire : « Unique intermédiaire entre persécuteurs et persécutés, son portrait 
n'est paradoxalement guère flatteur, même s'il refuse de servir Hynkel dans ses extrémités sanguinaires. Il succède au millionnaire de City Lights (les Lumières de la ville, 1931) appartenant lui aussi à la classe possédante, lui aussi un personnage à deux visages. Chaplin dessine avec ces deux figures l'image plutôt sévère d'une grande bourgeoisie opulente. La posture d'un tel personnage face au totalitarisme est un problème politique crucial: on peut penser que son indifférence initiale, voire son soutien (le sien et celui de sa classe sociale) n'est pas pour rien dans l'arrivée au pouvoir d'Hynkel ».

15 Je ne me trouve finalement en désaccord avec l'auteur que sur un point mineur, qui mérite malgré tout d'être signalé. Esquenazi écarte radicalement l'idée d'une fascination de Chaplin pour les dictateurs, motif il est vrai assez courant et parfois agaçant de la critique, qui s'appuie sur l'intérêt connu du cinéaste pour Napoléon et sur la mauvaise réputation de Chaplin directeur de troupe et patron d'entreprise. Le cinéaste a souvent revêtu le costume de Napoléon et il a longtemps caressé le projet de faire un film sur lui - que l'idée du Dictateur l'a finalement conduit à abandonner. Je persiste malgré tout à penser qu'il existe une dimension autoréférentielle et même autocritique dans le film, à laquelle ce qu'on sait du comportement quotidien du «tyran » du studio de La Brea apporte un crédit certain, et que cette dimension, loin de remettre en question le message politique de The Great Dictator, lui donne un surcroît de profondeur qui n'est pas sans annoncer les réflexions d'Arendt sur la «banalité du mal ». Le portrait de Hynkel n'est guère suspect de complaisance, c'est le moins que l'on puisse dire, mais dans les plis les plus « charlotiens » de son caractère et de son jeu (cette trace d'humanité que la comédie malgré tout lui donne, notamment quand il est confronté à dictateur plus dictateur que lui - Benzino Napaloni, dont le nom suggère qu'il est meilleur «Napoléon »), c'est aussi la tentation de tyrannie tapie en chacun de nous que le cinéaste donne à voir, et qu'il nous invite aussi à conjurer en nous en faisant rire. Le débat sur ce point reste naturellement ouvert.

16 J'ajoute pour finir que le livre d'Esquenazi, si concentré qu'il soit sur le film qu'il étudie, s'appuie sur une connaissance précise de l'ensemble de l'œuvre et de la vie de Chaplin. Quant à son iconographie, très bien choisie et d'une qualité de reproduction tout à fait exceptionnelle (car beaucoup de livres sur le cinéma n'en ont hélas plus les moyens), elle soutient en permanence la démonstration et contribue de façon non négligeable à l'intérêt et au plaisir de la lecture. 